\title{
Management of a photovoltaic-battery-based microgrid in a prosumer context
}

\author{
Mateus ALMEIDA BARBOSA ${ }^{1}$, Kübra GÜL ${ }^{1}$, Antoneta Iuliana BRATCU² and Iulian MUNTEANU² \\ ${ }^{1}$ ENSE3, Grenoble INP, 38031, Grenoble Cedex 1, France, Emails: \{mateus.almeida-barbosa, kubra.gul\}@grenoble-inp.fr \\ ${ }^{2}$ Univ. Grenoble Alpes, CNRS, Grenoble INP*, GIPSA-lab, 38402, Saint-Martin d'Hères Cedex, France \\ *Institute of Engineering Univ. Grenoble Alpes \\ Emails: \{antoneta.bratcu, iulian.munteanu\}@gipsa-lab.grenoble-inp.fr
}

\begin{abstract}
Prosumers are households that are both producers and consumers of electricity. In this paper, an Energy Management System (EMS) for a DC-microgrid-based prosumer is designed and simulated, whose aim is to coordinate operation of a photovoltaic (PV) system and a battery for supplying a DC load; interaction with the utility grid - modelled as an infinitepower source - is minimized, such that power is either injected or drawn only when strictly necessary. The PV system and the battery are paralleled on a common DC-link by means of a boost converter and a synchronous buck converter, respectively, while an inverter ensures connection to the utility grid. In order to design and simulate the individual components of the microgrid, MATLAB $^{\circledR} /$ Simulink $^{\circledR}$ tools were used. During the study, different operating scenarios were created with respect to the $\mathbf{P V}$ power, load and the state of charge (SoC) of the battery. Thus, the following operation modes were identified: following mode, discharge mode, charging mode - referring to the battery operating modes that depend of its SoC. The aim of the EMS is to ensure the requirements of a prosumer application and switch between these different modes. Simulation results have shown that the developed EMS can achieve effective coordination within the microgrid, and all goals are reached.
\end{abstract}

Keywords-prosumer; photovoltaic (PV); DC microgrid; Energy Management System (EMS); energy storage system

\section{INTRODUCTION}

A prosumer is a particular configuration of microgrid which consists basically of a household that acts both as producer and consumer of electricity [1], [2]. As technology advances, renewable energy sources are seen to increase their share in power production. Amongst the vast topics in which renewable generation takes part there is the microgrid, with a large range of applications. As renewable energy becomes more accessible to end users, as well as flexibilization becomes more latent in the energy transition the world is facing, microgrids benefit now from increased interest [3]-[5].

Amongst the renewable sources penetrating the microgrids, photovoltaic production provides good flexibility in terms of applicability, as it can be used for both large- and small-scale generation. The increase of prosumers who are using PV together with storage system brings advantages such as high efficiency and possibility to alleviate the renewable source's intermittency [6], [7]. Such PV-battery hybrid systems also play a vital role in managing peak demand and are, therefore, amongst the major stakeholders of the future utility grids. One of the solutions to increase the efficiency is an Energy Management System (EMS) which controls the power transfer between the elements of the microgrid [3], [8]. To increase the complexity, and possibly the range of applications for such a system, more elements could be added, such as a supercapacitor and a wind turbine [4], [5], for example. Technical aspect may also be completed by proposing business models and incentives for the prosumers [1].

In this paper design of an EMS for a DC microgrid-based prosumer is proposed. The chosen configuration of the studied microgrid can be seen in Figure 1 and it consists of: a photovoltaic (PV) system as renewable source, a battery unit as storage system, a DC load that models the consumer part and the AC grid. All these elements are connected in parallel to a common DC link by means of power electronics converters, namely: a synchronous-buck converter for the battery, a boost converter for the PV and an inverter for the AC grid.

This microgrid-based prosumer must operate according to several requirements, which are translated into control objectives as follows:

- the main aim is to maintain DC-link voltage (Vdc) as constant, which requires a closed-loop control system capable of dealing with disturbances;

- minimization of the net power flow through the AC grid, thus ensuring the prosumer autonomy as long as possible;

- control of power converters depending on battery SoC;

- Maximum Power Point Tracking (MPPT) implementation to maximize PV production.

By keeping the DC-link voltage constant, the DC sources of the system operate as current sources, whose currents are controlled by means of their associated converters. The inverter is also current-controlled. The current of each source is controlled by means of the duty cycle of the associated converter. In this way, a cascaded control structure is implemented, as suggested in Fig. 1. Here, variables noted as "Com1, 2, 3" and represented in blue denote current references sent by the upper-level EMS to the low-level current control loops. The low-level control consists of PI controllers used for synchronous buck converter and inverter in order to have zero steady-state error response due to the integral action and to 
guarantee the response time with proportional gain [9]. Consequently, their parameters were set depending on the imposed response time and overshoot. As concerns the MPPT, the Perturb and Observe (P\&O) control method was used to reach the maximum power point (MPP) of the PV array [10].

This paper is organized as follows. The following section will discuss all the steps towards the conception and validation of the proposed EMS. Section III will present the numerical simulation results obtained under representative scenarios, which validate the designed EMS's effectiveness. In Section IV conclusions regarding this work are drawn, as well as indications of future work using the presented configuration as base, are given.

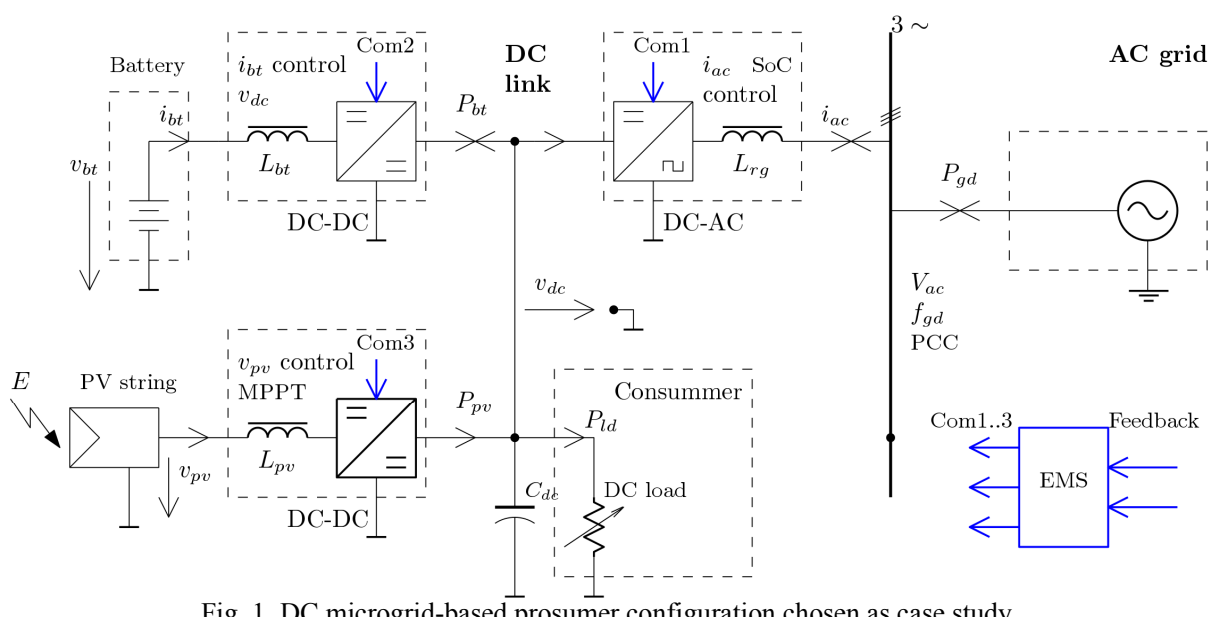

Fig. 1. DC microgrid-based prosumer configuration chosen as case study.

\section{ENERgy MANAGEMENT SyStem DESIGN}

\section{A. Goals and expected behavior}

The EMS is required to properly coordinate sources such as to accomplish the goals imposed by the prosumer configuration and operation. In the proposed cascaded control structure, the lower level acts mainly to fulfill the primary objective of maintaining the DC-link voltage constant, which is essential so the following steps can be set to accomplish the next goals.

The role of the EMS is then to provide current references for all the converters within the microgrid such that the DC load demand to be always guaranteed. To do so in this application the EMS must prioritize the PV production, which should always be operating at its maximum by means of an MPPT algorithm. The only situation the PV is not producing its maximum is in the case there is no DC load at the moment and the battery is already fully charged, and since there ought to be minimal interaction with the grid, the best decision is to disconnect the PV from the microgrid. This situation might change if considering a proper business model of the prosumer, that is, incentives related to its possibility of injecting power into the main grid. This case has not been considered here.

The battery will constantly act as a backup for the system, providing power to the grid when PV production is not enough and storing any excess otherwise, when production is higher than the demand. However, the battery will work within a certain range of operation, as it is not recommended to fully charge or discharge the battery [11]. This means that, when a predefined threshold is reached by the battery SoC when it is discharging, the EMS must command the immediate charging of the battery; and else, if the battery is charged beyond some other predefined limit of its SoC, the system must impose its immediate discharge.
For the above-mentioned behaviors to be correctly implemented, the EMS must be able to access the utility grid. In this context the grid is modelled as an infinite-power source, so it has the capacity to receive/inject as much power as needed from/to the prosumer. This means the EMS will demand power from the grid in case PV and battery productions are not enough to the DC load, or in case the battery must be charged, and the PV is not enough to do so. On the other hand, in case the battery must be discharged, or the PV production is too much, or a combination of both, the EMS will inject the power surplus into the grid.

\section{$B$. Low-level control loops}

This level refers to the modelling of each component of the system, followed by the design of their respective controllers. In this prosumer configuration the following components are modeled: the battery and its synchronous buck converter, the PV plant and its boost converter, the $\mathrm{AC}$ grid and the associated inverter, the DC load and the DC link.

PI controllers were then designed to govern the current of each converter of the system - thus, three tracking loops are implemented. The DC-link voltage, Vdc, must be kept constant despite disturbances coming from a priori unknown load variations - thus, its control loop is a regulation one. By assuming the DC-link voltage as constant, all sources can be modelled as current sources. Thus, they are current-controlled, meaning that the duty cycle of each converter will be altered so the current reference of each source to be tracked.

Concerning the PV array control, the chosen MPPT algorithm was the Perturb \& Observe. This algorithm is able to track in real time the position of MPP without any previous knowledge about its precise position. It receives information of current and voltage values of the PV array and calculates the 
instantaneous power value. Then a perturbation is caused on the voltage by means of adding or subtracting a fixed step and the new power is calculated. In case this new value is higher than the previous one, this increment in voltage is propagated; otherwise the algorithm changes its perturbation direction always towards the point of maximum power.

The current reference for the battery converter is used as control variable within the $\mathrm{Vdc}$ control loop, playing an essential role in maintaining its stability. The battery current control must quickly answer to any perturbation on the system, which happens mostly due to changes in the DC load and to the PV power variations. The current reference for the inverter is set by the EMS as one of its functions is to determine when to access the utility grid.

Regarding the AC part of the system, the modeling was based upon the Park transform [12], leading to $d$ and $q$ components. The $q$ component, being associated to the reactive power, it is kept at 0 , as here the goal is restricted to control the active power flow. The grid is modeled to work on $50 \mathrm{~Hz}$ and $400 \mathrm{~V}$ rms.

\section{Design methodology}

In order to design the desired EMS, the following methodology was adopted: first, the objectives the EMS must accomplish were determined, which means defining the scope of the system; secondly the operation scenarios were also established, then the EMS algorithm was conceived using a flow chart representation, and finally this algorithm was implemented on MATLAB ${ }^{\circledR} /$ Simulink $^{\circledR}$ so it could be tested and validated. The main objective of the EMS is to guarantee the coordination between the low-level control loops of the system in order to accomplish the previously described objectives. The EMS achieves this coordination by obtaining real-time information from the system, such as: measure of the PV power, measure of the power demanded by the load, the battery SoC estimation and the DC-link voltage measure. The battery $\mathrm{SoC}$ is here estimated by integrating its current $i_{\text {bat }}$ : $\operatorname{SoC}(t)=\operatorname{SoC}(0)+\int_{0}^{t} i_{b a t}(t) d t$, where $\operatorname{SoC}(0)$ is the initial
SoC, at time 0 . By using these values, the EMS changes accordingly the reference level of the inverter current.

To decide how to change the inverter current reference, a scenario analysis, which can be seen in Fig. 2, was made, followed by the algorithm description through a flow chart. In Fig. 2 Pdc denotes the power sensed on the DC link and Pac is the power exchanged with the $\mathrm{AC}$ grid, at the point of common coupling (PCC in Fig. 1).

The algorithm is based upon the remark that the reference of the battery current will follow alterations of the inverter current, according to power (current) equilibrium in the DC link node. Then, to command the charge or discharge of the battery according to its state of charge, the EMS sets the inverter current with the signal $(+$ or - ) with respect to the desired sense of power flow it wishes to impose.

Also, when defining the actual value of the reference, as one of the objectives is to minimize interaction with the grid, it is required that the grid will only be used in case the PV production is too high compared to the load and the excess of production must be directed to the grid, or when the demand cannot be met by the battery and PV productions together. Even in the last case, the battery will be set to work at its full capacity, during both discharge and charge, and the grid will only intervene when necessary. Finally, when the battery SoC is greater than the upper limit and there is no demand from the load, the PV is disconnected from the microgrid.

\section{Numerical implementation}

Once the desired behavior of the EMS was detailed, all cases were considered and the algorithm was established, the system is ready to be implemented on MATLAB ${ }^{\circledR} /$ Simulink $^{\circledR}$. When doing so, it was possible to identify three operating modes with respect to the battery status: follow mode, charging mode and discharging mode, respectively. This means that the algorithm has three branches that define how the inverter current reference will be set. In order to decide in which operation mode, the system currently is, it compares the battery SoC with some predefined threshold of a switch. These same patterns are repeated all across the diagram.

\begin{tabular}{|c|c|c|c|c|c|c|}
\hline \multicolumn{7}{|c|}{ Scenarios } \\
\hline Element & $\#$ & Pdc & SOCbat & Bat State & Pac & Comments \\
\hline \multirow{6}{*}{$\begin{array}{c}\text { Operation } \\
\text { State }\end{array}$} & 1 & Ppv $=$ Pload & High & Idle & Null & PV provides power for the load. \\
\hline & 2 & Ppv $=$ Pload & Low & Charging & Negative & $A C$ grid should charge battery. \\
\hline & 3 & Ppv>Pload & High & Idle & Positive & $\begin{array}{l}\text { PV production works on two states: MPPT or off. } \\
\text { Therefore the extra production must be sent to } \\
\text { the grid. }\end{array}$ \\
\hline & 4 & Ppv>Pload & Low & Charging & Null & PV charges Battery until reaches state (3) \\
\hline & 5 & Ppv<Pload & High & Discharging & Null & $\begin{array}{l}\text { In case Pbattery is not enough } \mathrm{Pac} \text { is needed } \\
2 \text { sub-cases }\end{array}$ \\
\hline & 6 & Ppv<Pload & Low & Charging & Negative & AC grid should charge battery. \\
\hline
\end{tabular}

Fig. 2. Analysis of operation scenarios 


\section{RESULTS UNDER REPRESENTATIVE SCENARIOS}

The obtained results must be analyzed as to assess the effectiveness of the developed EMS in fulfilling the imposed objectives. To this end, two testing scenarios have been defined. Thus, in the first scenario, the aim is to observe how the EMS fulfills its role in response to load variations, which are the main disturbance. Thus, the DC load variation has been shaped as a series of step variations, to challenge the whole domain of EMS operation. The second scenario is chosen to demonstrate how the system would behave in the absence of the PV, that is, during the night for instance.

Note that the follow mode is considered to be the default operation mode for the application. This is because the system operates mainly in residential applications with a robust storage system, meaning the battery capacity would be large enough so that charges and discharges occur slowly during a daily cycle, and would rarely operate in extreme conditions, that is, near the thresholds. For the same reason, it would mean that for a simulation time of even $1 \mathrm{~h}$ there would not happen significant changes in battery SoC, a crucial parameter to determine in which operation zone the system is. Therefore, the capacity of the battery was set to $300 \mathrm{Ah}$, a relatively low capacity, making the system more sensible to changes and allowing the results presented in the simulations to be conclusive.

The battery SoC must be assigned an initial value at each test. The minimum and maximum thresholds for it were set based on usual values from the literature, recommended to ensure the battery life span, and are respectively $30 \%$ and $90 \%$ [5], [6], [12]. This means that, in case the state of charge is under $30 \%$, the battery must be charged, and in case it is over $90 \%$ the battery must be discharged.

In the Simulink ${ }^{\circledR}$ implementation the current value of the state of charge is compared to the limiting values by means of a sum or a subtraction, the result of which is then put through a relay block producing a hysteresis effect; this avoids the oscillatory behavior around 0 and repetitive mode transitions back and forth. The battery will shift from follow to charging mode at a state of charge of $27,5 \%$ and will shift in the other sense at $32,5 \%$ as hysteresis limits are $-2,5 \%$ and $2,5 \%$. Similarly, the shift from follow to discharging mode will occur at $95 \%$ and in the other sense at $85 \%$, as in this case the hysteresis limits are $5 \%$ and $-5 \%$ respectively.

Without loss of generality, variations of the PV power are here not distinguished from those of the load itself, such that disturbances in both consumption and production are here treated globally, as coming from a so-called aggregated load. The aggregated load is the main responsible for generating such perturbations because it is influenced by two unpredictable variables: the irradiance and the demanded DC current. The first objective that must be attained by the EMS is keeping constant the voltage level at the DC link, which means the system must be able to quickly reject any perturbation.

\section{A. Scenario 1: complete overview between operation modes}

In this application the maximum power the PV is able to provide is $102.4 \mathrm{~kW}$. The DC load current variation domain is between 0 and $110 \mathrm{~A}$, varying in steps, as shown in Fig. 3. The grid here is seen as an infinite-power source. As for the battery, when it is in charging mode, a constant current of 100 A should by drawn, while when in discharging mode only an inferior limit is set, i.e., $100 \mathrm{~A}$. A random variation of the irradiation profile has been chosen, as seen in Fig. 4, in order to analyze if the system is able to remain stable even in the worst-case scenario of high-frequency changes.

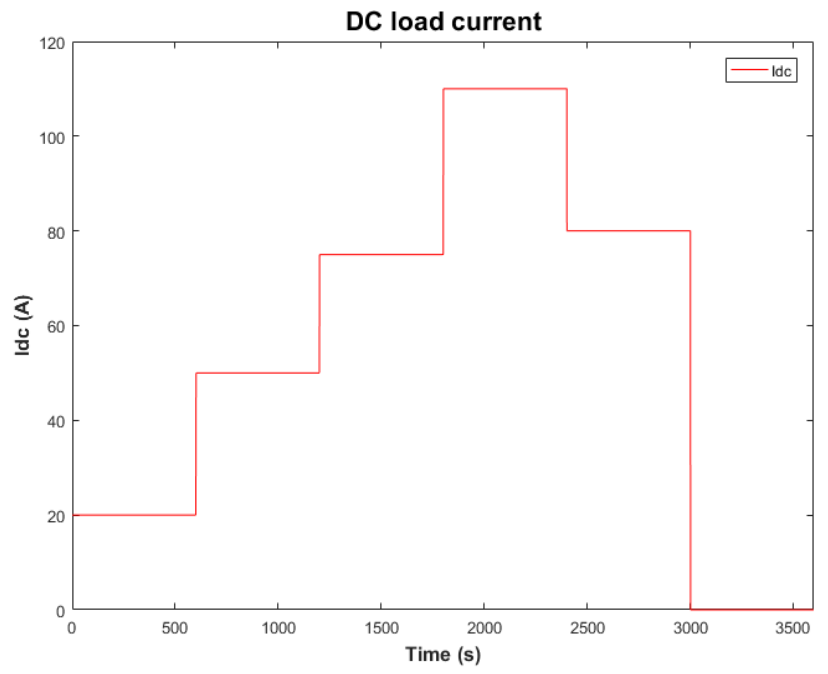

Fig. 3. DC load current (Idc) vs. time.

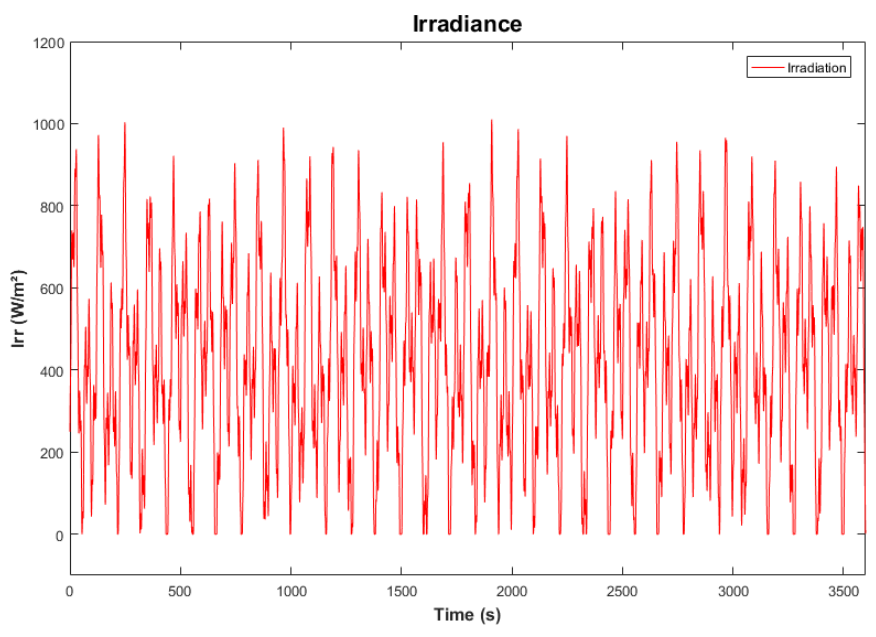

Fig. 4. Irradiance profile.

The simulation time span is of $3600 \mathrm{~s}$ ( 1 hour), sufficient to observe the three modes, starting from $97 \%$ as initial battery SoC, meaning the system operates in discharging mode. At this point the DC load current is set to $20 \mathrm{~A}$, as the battery discharges by providing a constant current of $80 \mathrm{~A}$. To balance this excess of current, the inverter acts by dispatching the other 60 A to the grid. Yet, as the PV is not needed in such context, the EMS automatically turns it off. This behavior can be observed in terms of power equilibrium in Fig. 5. To better understand what the graphs show, the generator convention is adopted. 


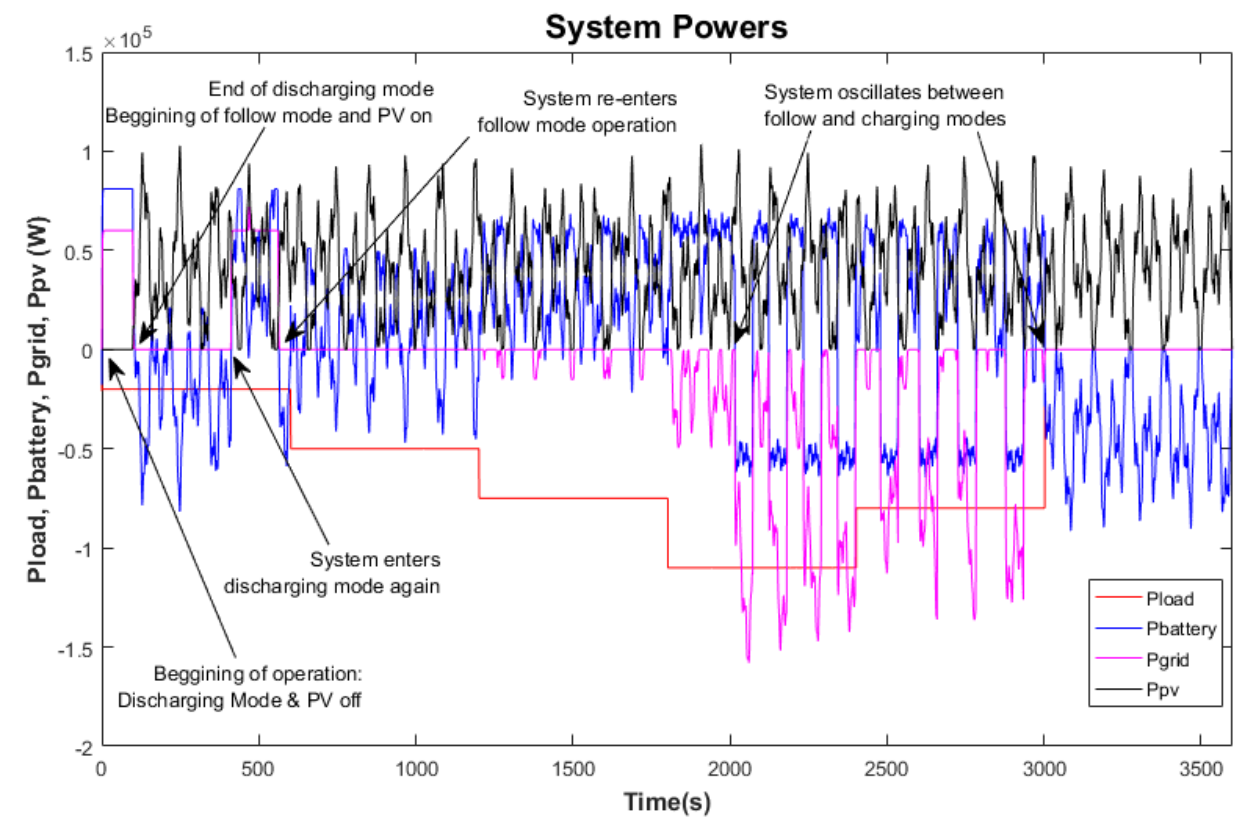

Fig. 5. Power values of interest vs. time, corresponding to first scenario.

Thus, currents of sources (battery, PV) are positive when these sources are providing power, whereas currents of loads are positive when loads are consuming power. The microgrid is seen as a source by the utility grid, so its current is positive when it is injected into the main grid. The DC-link voltage variation can be seen in Fig. 6, with a zoom in Fig. 7, allowing to observe the transients and dynamic performance. After $99 \mathrm{~s}$ the battery SoC reaches $85 \%$, meaning the system enters the follow mode. The PV is then turned on, causing a disturbance on Vdc, as shown in Fig. 6, and starts producing according to the irradiance profile curve.

When in follow mode, as the load is still relatively low in comparison to the PV production, interaction with the grid is no longer needed and battery receives the exceeding solar production charging itself until $95 \%$ of SoC, at around $412 \mathrm{~s}$.

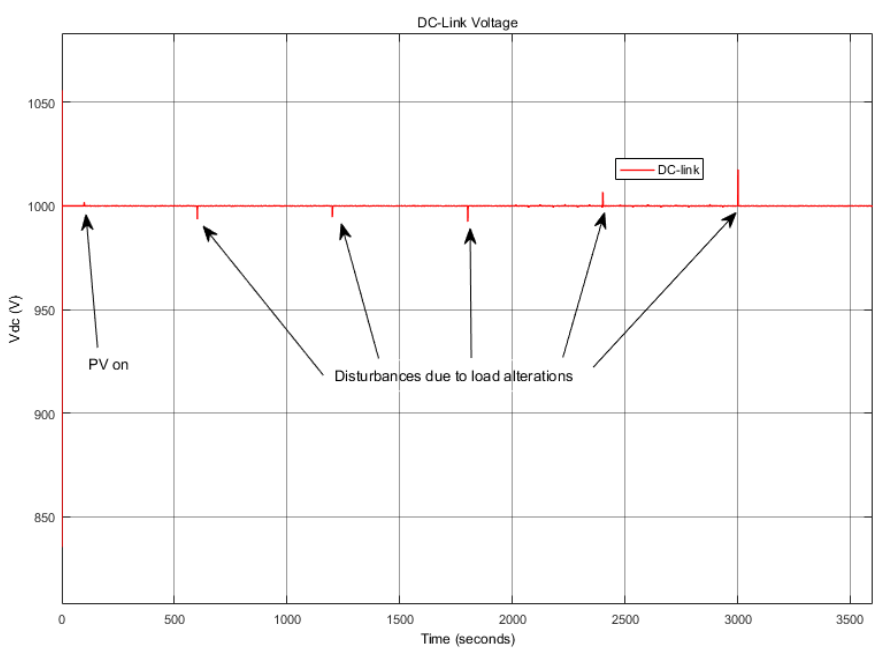

Fig. 6. DC-link voltage (Vdc) vs. time, in the first scenario.

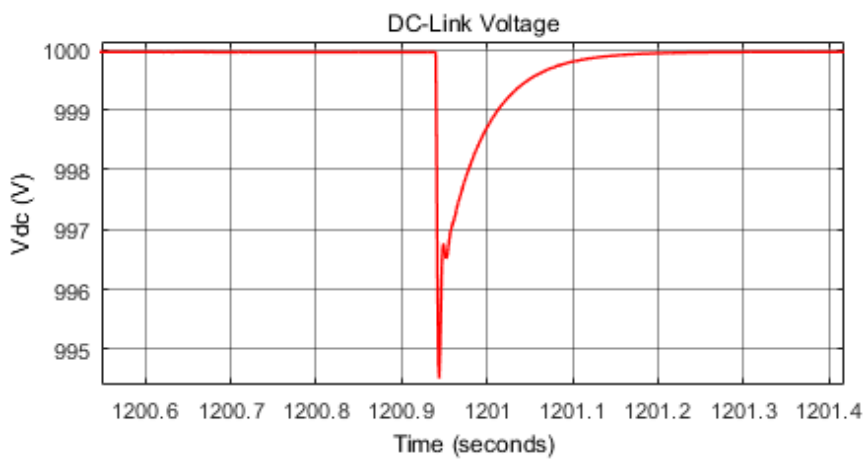

Fig. 7. Zoom of Vdc evolution around 1200 seconds, in the first scenario.

Then the battery re-enters discharging and again the inverter is solicited to inject current into the grid. Once the battery SoC reaches $85 \%$, the system is again in follow mode and battery begins to be charged, but at $600 \mathrm{~s}$ the load is increased to $50 \mathrm{~A}$, which in return demands the battery to provide power to the load depending on the level of the PV production. This process slowly discharges the battery. Also, the load increase is reflected as a perturbation on Vdc (the second spike visible in Fig. 6), which is rejected due to EMS.

Further on, at around $1200 \mathrm{~s}$, the load is again increased, this time to $75 \mathrm{~A}$, generating yet another perturbation, and the discharge rate of the battery increases as its use is primary to demand power from the utility grid. This is seen as the third disturbance in Fig. 6, which is again quickly rejected by the system, as expected. In the zoom in Fig. 7 the Vdc response to the perturbation can be seen in more detail, showing that the system returns to the nominal point after approximately $0.3 \mathrm{~s}$.

The last increase occurs at $1800 \mathrm{~s}$ and represents a demand of $110 \mathrm{~A}$. This goes beyond the maximal production from the PV and, associated with the high-frequency variability of the 
irradiance, even the addition of the battery is not enough at some moments, which will then force the system to draw current from the grid. This demand increases still at $2000 \mathrm{~s}$ when the battery reaches charging mode for the first time, meaning not only the load must be supplied but also the battery must be charged until acceptable levels in order to preserve an operational level of its SoC. What then follows is that every time the battery arrives in follow mode it discharges again while supplying to the system, and then it is charged again. Even at $2400 \mathrm{~s}$, when the load is reduced to $80 \mathrm{~A}$, the same behavior can still be observed. This cycle breaks at $3000 \mathrm{~s}$, when the load drops at $0 \mathrm{~A}$ and the battery continues to be charged even after it enters follow mode, as PV is still producing more than needed. Also, from this point on the inverter current returns to $0 \mathrm{~A}$, ensuring that the interaction with the grid is minimized.

It can be concluded from this scenario that all system parts are correctly coordinated by the EMS. All the imposed objectives are achieved.

\section{B. Scenario 2: Special operation under no PV production}

This second testing scenario is intended to simulate an operation during the night when irradiance levels would drop to zero, thus there would not be production from the PV. The load was initially set to $50 \mathrm{~A}$, then dropped at $0 \mathrm{~A}$ at $500 \mathrm{~s}$, in the middle of the $1000 \mathrm{~s}$ total simulation time, as to simulate a low demand period during the night (Fig. 8). Again, the results verify that the goals have been fulfilled. Load is supplied by the battery until it reaches its threshold and enters charging mode, requiring current from the grid. Once the battery is in follow mode and there is no demand from the load, all the power flows remain 0 and $\mathrm{Vdc}$ remains constant.

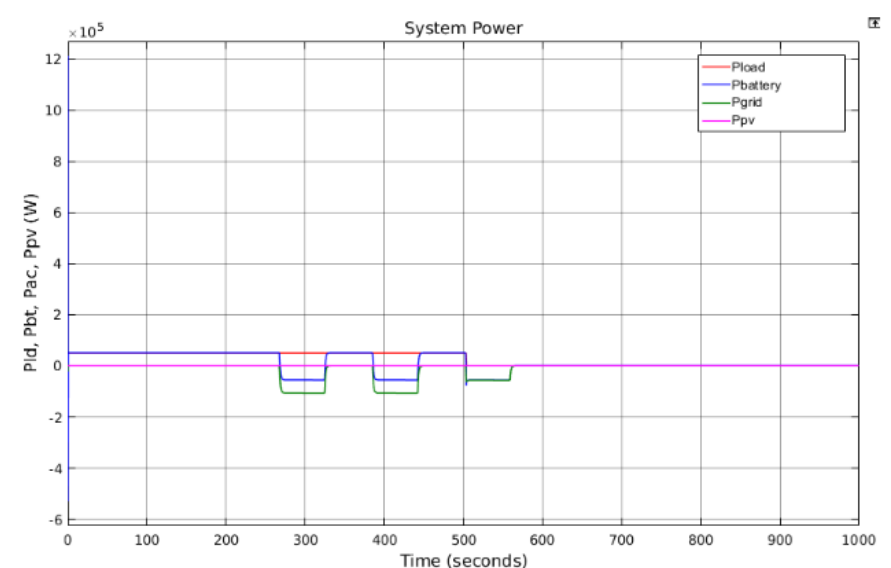

Fig. 8. Power values of interest in the second scenario.

\section{CONCLUSION AND FUTURE WORK}

In this paper, a DC-microgrid Energy Management System (EMS) has been proposed to coordinate operation of a photovoltaic (PV) system and a battery in supplying a DC load in a prosumer context. To this end, interaction with the utility grid - modelled as an infinite-power source - takes only place when strictly necessary. PV system and the battery are paralleled on a common DC-link by means of a boost converter and a synchronous buck converter, respectively, while an inverter ensures connection to the utility grid. MATLAB $^{\circledR} /$ Simulink $^{\circledR}$ numerical simulations have illustrated the effectiveness of the proposed EMS under representative scenarios, defined with respect to the PV power, load and the battery state of charge (SoC). The EMS has proven its ability to ensure the requirements of a prosumer application and switch between the different operating modes.

Future work could explore opportunity of defining business models for prosumers, like, for example, understanding energy tariffs depending on the hour of the day and the demand and how this can be translated into constraints to the EMS and then optimized so the profitability could be increased. This type of study can be of fundamental importance when a potential consumer decides between whether or not taking up his own renewable production. This could also be the case of comparing potential revenues between different combinations of renewable sources and storage systems.

\section{REFERENCES}

[1] I. Ranaweera, O.-M. Midtgard, and M. Korpas, "Distributed control scheme for residential battery energy storage units coupled with PV systems," Renewable Energy, vol. 113, pp. 1099-1110, 2017.

[2] R. Khalipour and A. Vassalo, "Planning and operation scheduling of PV-battery systems: A novel methodology," Renewable and Sustainable Energy Reviews, vol. 53, pp. 194-208, 2016.

[3] Z. Yang, Z. Chen, L. Guo, Y. Chen, X. Huang, and Y. Wang, "Design Techniques of Distributed Photovoltaic/Energy Storage System," in 2017 20th International Conference on Electrical Machines and Systems (ICEMS), DOI 10.1109/ICEMS.2017.8056360.

[4] S. Bacha, I. Munteanu, and A.I. Bratcu, Power Electronic Converters Modeling and Control with Case Studies. Springer, 2014.

[5] L. Ma and Y. Xiaobo, "A Novel Series PV Energy Storage System," in IECON 2016 - 42nd Annual Conference of the IEEE Industrial Electronics Society, pp. 3184-3189, DOI 10.1109/IECON.2016.7793606.

[6] A. Aktas, K. Erhan, S. Ozdemir, and E. Ozdemir, "Dynamic energy management for photovoltaic power system including hybrid energy storage in smart grid applications," Energy, vol. 162, pp. 72-82, 2018.

[7] L. Ming-yu, Y. Zi-long, W. Yi-bo, and X. Hong-hua, "Study on Control Strategy of Energy Storage System in Photovoltaic Microgrid," in 2014 International Conference on Power System Technology (POWERCON 2014), DOI 10.1109/POWERCON.2014.6993558.

[8] U. Sangpanich, "A Novel Method of Decentralized Battery Energy Management for Stand-Alone PV-Battery Systems," in 2014 IEEE PES Asia-Pacific Power and Energy Engineering Conference (APPEEC), DOI 10.1109/APPEEC.2014.7066077.

[9] K. Astrom and T. Hägglund, PID Controllers: theory, design and tunning. International Society of Automation, 1994.

[10] R. Rakhmawati, Irianto, and B.W. Dionova, "Design and Implementation of Hybrid Energy System for DC Load Applications," in 2017 International Seminar on Application for Technology of Information and Communication (iSemantic), DOI 10.1109/ISEMANTIC.2017.8251853.

[11] R. Zafara, A. Mahmoodb, S. Razzaq, W. Ali, and U. Naeem, "Prosumer based energy management and sharing in smart grid," Renewable and Sustainable Energy Reviews, vol. 82, Part 1, pp. 1675-1684, 2018.

[12] T. Rout, A. Chowdhury, D.M. Maharana, and P.S. Samal, "Analysis of Energy Management System for Photovoltaic System with Battery and Supercapacitor using Fuzzy Logic Controller," in IEEE International Conference on Technologies for Smart-City Energy Security and Power (ICSESP-2018), DOI 10.1109/ICSESP.2018.8376737. 